\title{
On the Periodic Auto-Oscillations of an Electric Circuit with Periodic Imperfections on Its Variables
}

\author{
M. Teresa de Bustos ${ }^{1}$, Miguel A. López ${ }^{2, *}$ and Raquel Martínez ${ }^{2}$ \\ ${ }^{1}$ Departamento de Matemática Aplicada. Universidad de Salamanca, Casas del Parque, 2, 37008-Salamanca, Spain \\ ${ }^{2}$ Departamento de Matemáticas, Universidad de Castilla-La Mancha, Campus de Cuenca, 16071-Cuenca, Spain
}

Received: 20 Feb. 2013, Revised: 18 Jun. 2013, Accepted: 21 Jun. 2013

Published online: 1 Nov. 2013

\begin{abstract}
The aim of the present paper is to study the periodic auto-oscillations of an electric circuit with periodic imperfections on its variables composed by three condensers, one of them without charge, and two bobbins. We model this system by the Lagrangian approach using the morphology of the Hill problem and the main tool used for proving the results is the averaging theory of dynamical systems.
\end{abstract}

Keywords: Periodic solutions, electric circuit, averaging theory, dynamical systems

\section{Introduction and statement of the main results}

We consider a dynamical systems consistent in an electric circuit composed by by three condensers and two bobbins such that its variables have periodic imperfections, i.e. our model is a perturbation of the ideal circuit, see Fig. 1. The aim of our work is to study the periodic orbits, i.e. auto-oscillations, produced by the system. For doing this we shall use the averaging theory of dynamical systems, see Appendix for more details on it. We have been inspired by other works where these techniques have been used for studying other perturbed dynamics problems, see for instance $[2,3,4,5,6,7,8,9,10]$.

We consider the Lagrangian formulation of the circuit, using the morphology of the Hill problem,

$$
\mathscr{L}=\frac{1}{2}\left(L_{1} \dot{q}_{1}^{2}+L_{2} \dot{q}_{2}^{2}\right)-\frac{q_{1}^{2}}{\bar{c}_{1}}-\frac{q_{2}^{2}}{\bar{c}_{2}}-\frac{\left(q_{1}+q_{2}\right)^{2}}{\bar{c}}
$$

where $q_{i}$ are the charges and $c_{i}$ are the capacities of the condensers, $i \in\{1,2\}$. The variables $L_{i}, i \in\{1,2\}$, represent the the auto-inductions of the bobbins. $c$ represents the capacity of a third condenser without charge.

If we make the change of variable:

$$
x=\sqrt{L_{1}} q_{1}, \quad y=\sqrt{L_{2}} q_{2},
$$

we obtain:

$$
\mathscr{L}=\frac{1}{2}\left(\dot{x}^{2}+\dot{y}^{2}\right)-\frac{1}{c_{1}} x^{2}-\frac{1}{c_{2}} y^{2}-\frac{1}{c} x y
$$

being, for $i=1,2$,

$$
\frac{1}{c_{i}}=\frac{1}{L_{i} \bar{c}_{i}}+\frac{1}{L_{i} \bar{c}}
$$

and

$$
\frac{1}{c}=\frac{2}{\bar{c} \sqrt{L_{1} L_{2}}}
$$

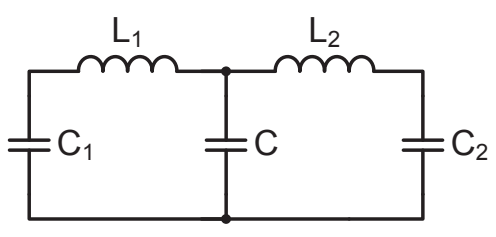

Fig. 1: Circuit

Using the Legendre transformation, we obtain the Hamiltonian:

$$
\mathscr{H}=\frac{1}{2}\left(\dot{x}^{2}+\dot{y}^{2}\right)+\frac{1}{c_{1}} x^{2}+\frac{1}{c_{2}} y^{2}+\frac{1}{c} x y
$$

\footnotetext{
*Corresponding author e-mail: mangel.lopez@uclm.es
} 
Thus, our perturbed model has the following form:

$$
\begin{aligned}
& \dot{x}=p_{1} \\
& \ddot{x}+\frac{2}{c_{1}} x+\frac{1}{c} y=\varepsilon F_{1}(t, x, \dot{x}, y, \dot{y}) \\
& \dot{y}=p_{2} \\
& \ddot{y}+\frac{1}{c} x+\frac{2}{c_{2}} y=\varepsilon F_{2}(t, x, \dot{x}, y, \dot{y})
\end{aligned}
$$

where $p_{1}=\frac{\partial \mathscr{L}}{\partial \dot{x}}$ and $p_{2}=\frac{\partial \mathscr{L}}{\partial \dot{y}}$. The dot denotes the derivative with respect to the time $t$, the parameter $\varepsilon$ is small and the smooth functions $F_{1}$ and $F_{2}$, in general, are periodic functions in the variable $t$ and in resonance $p: q$ with some of the periodic solutions for $\varepsilon=0$, being $p$ and $q$ positive integers relatively prime.

The objective of this paper is to provide, using the averaging theory, a system of nonlinear equations whose simple zeros provide periodic solutions of the differential system (1). In order to present our results we need some preliminary definitions and notation.

The unperturbed system with four differential equations of second order

$$
\begin{aligned}
\dot{x} & =p_{1} \\
\ddot{x} & =-\frac{2}{c_{1}} x-\frac{1}{c} y \\
\dot{y} & =p_{2} \\
\ddot{y} & =-\frac{1}{c} x-\frac{2}{c_{2}} y
\end{aligned}
$$

written as a differential system of first order in the four variables $\left(X_{1}=x, X_{2}=\dot{x}, X_{3}=y, X_{4}=\dot{y}\right)$,

$$
\begin{aligned}
& \dot{X}_{1}=X_{2} \\
& \dot{X}_{2}=-\frac{2}{c_{1}} X_{1}-\frac{1}{c} X_{3} \\
& \dot{X}_{3}=X_{4} \\
& \dot{X}_{4}=-\frac{1}{c} X_{1}-\frac{2}{c_{2}} X_{3}
\end{aligned}
$$

has a unique singular point at the origin with eigenvalues

$$
\pm \omega_{1} i, \quad \pm \omega_{2} i
$$

which are the roots of the polynomial

$$
4 c^{2}-c_{1} c_{2}+2 c^{2} c_{1} \omega^{2}+2 c^{2} c_{2} \omega^{2}+c^{2} c_{1} c_{2} \omega^{4}
$$

where $c, c_{1}$ and $c_{2} \in \mathbb{R}^{+}$and $c_{1} c_{2}<4 c^{2}$.

The frequencies $\omega_{i}$ are given by

$$
\begin{aligned}
& \omega_{1}=\frac{\sqrt{c c_{1} c_{2}\left(c\left(c_{1}+c_{2}\right)+\sqrt{c^{2}\left(c_{1}-c_{2}\right)^{2}+c_{1}^{2} c_{2}^{2}}\right)}}{c c_{1} c_{2}}, \\
& \omega_{2}=\frac{\sqrt{c c_{1} c_{2}\left(c\left(c_{1}+c_{2}\right)-\sqrt{c^{2}\left(c_{1}-c_{2}\right)^{2}+c_{1}^{2} c_{2}^{2}}\right)}}{c c_{1} c_{2}}
\end{aligned}
$$

Note that $\omega_{1}^{2}-\omega_{2}^{2}=\frac{2 \sqrt{c^{2}\left(c_{1}-c_{2}\right)^{2}+c_{1}^{2} c_{2}^{2}}}{c c_{1} c_{2}}$.

As usual we define that the ratio of the two frequencies $\omega_{i}$ and $\omega_{j}$ is non-resonant with $\pi$ if $\omega_{i} \pi / \omega_{j}$ is not a rational number, $i \neq j$.

System (2) in the phase space $(x, \dot{x}, y, \dot{y})$ has two planes passing through the origin filled of periodic solutions with the exception of the origin. These periodic solutions have periods $T_{1}=2 \pi / \omega_{1}$ and $T_{2}=2 \pi / \omega_{2}$, according they belong to the plane associated to the eigenvectors with eigenvalues $\pm \omega_{1} i$ or $\pm \omega_{2} i$, respectively. We shall study which of these periodic solutions persist for the perturbed system (1) when the parameter $\varepsilon$ is sufficiently small and the perturbed functions $F_{i}$, for $i=1,2$, have period either $p T_{1} / q$ or $p T_{2} / q$, where $p$ and $q$ are positive integers relatively prime.

We define the constants $\phi$ and $\rho$ by

$$
\phi=\frac{c_{2}-c_{1}}{c_{1} c_{2}}, \quad \rho=\omega_{1}^{2}-\omega_{2}^{2},
$$

and the functions:

$$
\begin{aligned}
\mathscr{G}_{1}^{1}\left(X_{1}^{0}, X_{2}^{0}\right) & =\frac{1}{p T_{1}} \int_{0}^{p T_{1}} \cos \left(\omega_{1} t\right) F_{1}^{*}(t) d t, \\
\mathscr{G}_{1}^{2}\left(X_{1}^{0}, X_{2}^{0}\right) & =\frac{1}{p T_{1}} \int_{0}^{p T_{1}} \sin \left(\omega_{1} t\right) F_{1}^{*}(t) d t
\end{aligned}
$$

where

$$
F_{1}^{*}(t)=\frac{1}{4 c \rho}\left[2 F_{1}+c(\rho-2 \phi) F_{2}\right]
$$

with

$$
F_{i}=F_{i}\left(\sigma_{1}^{1}(t), \sigma_{1}^{2}(t), \sigma_{1}^{3}(t), \sigma_{1}^{4}(t)\right), i=1,2,
$$

and

$$
\begin{aligned}
\sigma_{1}^{1}(t) & =\frac{-2 c c_{2} \omega_{1}}{c_{2}+c^{2}(\rho-2 \phi)}\left(X_{2}^{0} \cos \left(\omega_{1} t\right)-X_{1}^{0} \sin \left(\omega_{1} t\right)\right) \\
\sigma_{1}^{2}(t) & =c(\rho+2 \phi)\left(X_{1}^{0} \cos \left(\omega_{1} t\right)+X_{2}^{0} \sin \left(\omega_{1} t\right)\right) \\
\sigma_{1}^{3}(t) & =\frac{-2}{\omega_{1}}\left(X_{2}^{0} \cos \left(\omega_{1} t\right)-X_{1}^{0} \sin \left(\omega_{1} t\right)\right) \\
\sigma_{1}^{4}(t) & =2\left(X_{1}^{0} \cos \left(\omega_{1} t\right)+X_{2}^{0} \sin \left(\omega_{1} t\right)\right)
\end{aligned}
$$

A zero $\left(X_{1}^{0 *}, X_{2}^{0 *}\right)$ of the nonlinear system

$$
\mathscr{G}_{1}^{1}\left(X_{1}^{0}, X_{2}^{0}\right)=0, \quad \mathscr{G}_{1}^{2}\left(X_{1}^{0}, X_{2}^{0}\right)=0,
$$

such that

$$
\left.\operatorname{det}\left(\frac{\partial\left(\mathscr{G}_{1}^{1}, \mathscr{G}_{1}^{2}\right)}{\partial\left(X_{1}^{0}, X_{2}^{0}\right)}\right)\right|_{\left(X_{1}^{0}, X_{2}^{0}\right)=\left(X_{1}^{0 *}, X_{2}^{0 *}\right)} \neq 0
$$

is called a simple zero of system (4).

The statement of our main result on the periodic solutions of the differential system (1) which bifurcate 
from the periodic solutions of period $T_{1}$ of the unperturbed system traveled $p$ times is the following.

Theorem 1.1. Let $p$ and $q$ be positive integers relatively prime and assume that the smooth functions $F_{1}$ and $F_{2}$ of the equations of motion of (1) are periodic in the variable $t$ of period $p T_{1} / q$. We assume that the ratio of the frequencies $\omega_{2} / \omega_{1}$ is not resonant with $\pi$. Then for $\varepsilon \neq 0$ sufficiently small and for every simple zero $\left(X_{1}^{0 *}, X_{2}^{0 *}\right) \neq(0,0)$ of the nonlinear system (4), the perturbed system (1) has a periodic solution $(x(t, \varepsilon), y(t, \varepsilon))$ tending to the periodic solution $(x(t), y(t))=\left.\left(\sigma_{1}^{1}(t), \sigma_{1}^{3}(t)\right)\right|_{\left(X_{1}^{0}, X_{2}^{0}\right)=\left(X_{1}^{0 *}, X_{2}^{0 *}\right)} \quad$ of the unperturbed system (2) traveled $p$ times.

Theorem 1.1 is proved in section 2. Its proof is based in the averaging theory for computing periodic solutions, see Appendix I for more details on this technique.

An application of Theorem 1.1 is presented in the following corollary, which will be proved in section 3 .

Corollary 1.2. Let $F_{1}(t, x, \dot{x}, y, \dot{y})=\dot{x}^{2}+\dot{y}^{2}$ and $F_{2}(t, x, \dot{x}, y, \dot{y})=\sin \left(\omega_{1} t\right)\left(1-\dot{x}^{2}\right)+\cos \left(\omega_{1} t\right)(x-\dot{y})$ be perturbed functions and that the ratio of the frequencies $\omega_{2} / \omega_{1}$ is not resonant with $\pi$. Then the system (1) for $\varepsilon \neq 0$ sufficiently small has two periodic solutions $(x(t, \varepsilon), y(t, \varepsilon))$ tending to the two periodic solutions $(x(t), y(t))=\left.\left(\sigma_{1}^{1}(t), \sigma_{1}^{3}(t)\right)\right|_{\left(X_{1}^{0}, X_{2}^{0}\right)=\left(X_{1}^{*}, X_{2}^{*}\right)} \quad$ and $(x(t), y(t))=\left.\left(\sigma_{1}^{1}(t), \sigma_{1}^{3}(t)\right)\right|_{\left(X_{1}^{0}, X_{2}^{0}\right)=\left(X_{3}^{*}, X_{4}^{*}\right)}$ of (2) when $\varepsilon \rightarrow 0$, where

$$
\begin{array}{r}
\left(X_{1}^{*}, X_{2}^{*}\right)=\left(0, \pm \sqrt{\frac{1-c^{2} \phi(\rho-2 \phi)}{3}}\right) \text { and } \\
\left(X_{3}^{*}, X_{4}^{*}\right)=\left( \pm \sqrt{1-c^{2} \phi(\rho-2 \phi)}, 0\right) .
\end{array}
$$

Corollary 1.2 will be proved in section 3 .

Now we define the functions:

$$
\begin{aligned}
\mathscr{G}_{2}^{1}\left(X_{5}^{0}, X_{6}^{0}\right) & =\frac{1}{p T_{2}} \int_{0}^{p T_{2}} \cos \left(\omega_{2} t\right) F_{3}^{*}(t) d t \\
\mathscr{G}_{2}^{2}\left(X_{5}^{0}, X_{6}^{0}\right) & =\frac{1}{p T_{2}} \int_{0}^{p T_{2}} \sin \left(\omega_{2} t\right) F_{3}^{*}(t) d t
\end{aligned}
$$

where

$$
F_{3}^{*}(t)=\frac{1}{4 c \rho}\left[-2 F_{1}+c(\rho+2 \phi) F_{2}\right]
$$

with

$$
F_{i}=F_{i}\left(\sigma_{2}^{1}(t), \sigma_{2}^{2}(t), \sigma_{2}^{3}(t), \sigma_{2}^{4}(t)\right), i=1,2
$$

and

$$
\begin{aligned}
\sigma_{2}^{1}(t) & =\frac{-2 c c_{2} \omega_{2}}{c_{2}-c^{2}(\rho-2 \phi)}\left(X_{6}^{0} \cos \left(\omega_{2} t\right)-X_{5}^{0} \sin \left(\omega_{2} t\right)\right) \\
\sigma_{2}^{2}(t) & =-c(\rho-2 \phi)\left(X_{5}^{0} \cos \left(\omega_{2} t\right)+X_{6}^{0} \sin \left(\omega_{2} t\right)\right) \\
\sigma_{2}^{3}(t) & =\frac{-2}{\omega_{2}}\left(X_{6}^{0} \cos \left(\omega_{2} t\right)-X_{5}^{0} \sin \left(\omega_{2} t\right)\right) \\
\sigma_{2}^{4}(t) & =2\left(X_{5}^{0} \cos \left(\omega_{2} t\right)+X_{6}^{0} \sin \left(\omega_{2} t\right)\right)
\end{aligned}
$$

Consider the nonlinear system

$$
\mathscr{G}_{2}^{1}\left(X_{5}^{0}, X_{6}^{0}\right)=0, \quad \mathscr{G}_{2}^{2}\left(X_{5}^{0}, X_{6}^{0}\right)=0 .
$$

The statement of our main result on the periodic solutions of the differential system (1) which bifurcate from the periodic solutions of period $T_{2}$ of the unperturbed system traveled $p$ times is the following.

Theorem 1.3. Let $p$ and $q$ be positive integers relatively prime and assume that the smooth functions $F_{1}$ and $F_{2}$ of the equations of motion of (1) are periodic in the variable $t$ of period $p T_{2} / q$. We assume that the ratio of the frequencies $\omega_{2} / \omega_{1}$ is not resonant with $\pi$. Then for $\varepsilon \neq 0$ sufficiently small and for every simple zero $\left(X_{5}^{0 *}, X_{6}^{0 *}\right) \neq(0,0)$ of the nonlinear system (5), the perturbed system (1) has a periodic solution $(x(t, \varepsilon), y(t, \varepsilon))$ tending to the periodic solution $(x(t), y(t))=\left.\left(\sigma_{2}^{1}(t), \sigma_{2}^{3}(t)\right)\right|_{\left(X_{5}^{0}, X_{6}^{0}\right)=\left(X_{5}^{0 *}, X_{6}^{0 *}\right)} \quad$ of the unperturbed system (2) traveled $p$ times.

Theorem 1.3 is proved in section 2 .

In the next corollary an application of Theorem 1.3 is given.

Corollary 1.4. Let $F_{1}(t, x, \dot{x}, y, \dot{y})=\sin \left(\omega_{2} t\right)(1+x+y)$ and $F_{2}(t, x, \dot{x}, y, \dot{y})=1+\dot{x}$ be perturbed functions and that the ratio of the frequencies $\omega_{2} / \omega_{1}$ is not resonant with $\pi$. Then the system (1) for $\varepsilon \neq 0$ sufficiently small has one periodic solution $(x(t, \varepsilon), y(t, \varepsilon))$ tending to the periodic solution $(x(t), y(t))=\left.\left(\sigma_{2}^{1}(t), \sigma_{2}^{3}(t)\right)\right|_{\left(X_{5}^{0}, X_{6}^{0}\right)=\left(X_{5}^{0 *}, X_{6}^{0 *}\right)}$ of (2) when $\varepsilon \rightarrow 0$, given by $\left(X_{5}^{0 *}, X_{6}^{0 *}\right)=\left(0, \frac{-1}{2}\right)$.

Corollary 1.4 will be proved in section 3 .

\section{Proof of the Theorems 1.1 and 1.3}

Introducing the variables $\left(X_{1}, X_{2}, X_{3}, X_{4}\right)=(x, \dot{x}, y, \dot{y})$ we can write the differential system (1) as a first-order differential system defined in $\mathbb{R}^{4}$ in the following form

$$
\begin{aligned}
& \dot{X}_{1}=X_{2} \\
& \dot{X}_{2}=-\frac{2}{c_{1}} X_{1}-\frac{1}{c} X_{3}+\varepsilon F_{1}\left(X_{1}, X_{2}, X_{3}, X_{4}\right) \\
& \dot{X}_{3}=X_{4} \\
& \dot{X}_{4}=-\frac{1}{c} X_{1}-\frac{2}{c_{2}} X_{3}+\varepsilon F_{2}\left(X_{1}, X_{2}, X_{3}, X_{4}\right)
\end{aligned}
$$


Note that the differential system (6) when $\varepsilon=0$ is equivalent to the differential system (2), called simply in what follows the unperturbed system. When $\varepsilon \neq 0$ we called it the perturbed system.

The change of variables

$$
\mathrm{x}=\left(x_{1}, x_{2}, x_{3}, x_{4}\right) \rightarrow \mathrm{X}=\left(X_{1}, X_{2}, X_{3}, X_{4}\right)
$$

given by

$$
\mathrm{X}=B \mathrm{x}
$$

with

$$
B=\left(\begin{array}{cccc}
0 & \frac{-2 c c_{2} \omega_{1}}{c_{2}+c^{2}(\rho-2 \phi)} & 0 & \frac{-2 c c_{2} \omega_{2}}{c_{2}-c^{2}(\rho+2 \phi)} \\
c(\rho+2 \phi) & 0 & -c(\rho-2 \phi) & 0 \\
0 & \frac{-2}{\omega_{1}} & 0 & \frac{-2}{\omega_{2}} \\
2 & 0 & 2 & 0
\end{array}\right)
$$

writes the linear part of the differential system (6) in its real Jordan normal form, and this system in the new variables $\left(x_{1}, x_{2}, x_{3}, x_{4}\right)$ becomes

$$
\begin{aligned}
& \dot{x}_{1}=\omega_{1} x_{2}+\varepsilon F_{1}^{*} \\
& \dot{x}_{2}=-\omega_{1} x_{1}+\varepsilon F_{2}^{*} \\
& \dot{x}_{3}=\omega_{2} x_{4}+\varepsilon F_{3}^{*} \\
& \dot{x}_{4}=-\omega_{2} x_{3}+\varepsilon F_{4}^{*}
\end{aligned}
$$

where

$$
\begin{aligned}
F_{1}^{*} & =\frac{1}{4 c \rho}\left[2 F_{1}+c(\rho-2 \phi) F_{2}\right] \\
F_{2}^{*} & =0 \\
F_{3}^{*} & =\frac{1}{4 c \rho}\left[-2 F_{1}+c(\rho+2 \phi) F_{2}\right] \\
F_{4}^{*} & =0
\end{aligned}
$$

with $F_{i}=F_{i}\left(\sigma^{1}, \sigma^{2}, \sigma^{3}, \sigma^{4}\right)$, and

$$
\begin{aligned}
\sigma^{1} & =-\frac{2 c c_{2} \omega_{1}}{c_{2}+c^{2}(\rho-2 \phi)} x_{2}-\frac{2 c c_{2} \omega_{2}}{c_{2}-c^{2}(\rho+2 \phi)} x_{4} \\
\sigma^{2} & =c(\rho+2 \phi) x_{1}-c(\rho-2 \phi) x_{3} \\
\sigma^{3} & =-\frac{2}{\omega_{1}} x_{2}-\frac{2}{\omega_{2}} x_{4} \\
\sigma^{4} & =2 x_{1}+2 x_{3}
\end{aligned}
$$

Now, in the following lemma we characterize the periodic orbits of the unperturbed system as a first step for proving Theorems 1.1 and 1.3.

Lemma 2.1. The periodic solutions $\left(x_{1}(t), x_{2}(t), x_{3}(t), x_{4}(t)\right)$ of the differential system (8) with $\varepsilon=0$ are

$\left(X_{1}^{0} \cos \left(\omega_{1} t\right)+X_{2}^{0} \sin \left(\omega_{1} t\right), X_{2}^{0} \cos \left(\omega_{1} t\right)-X_{1}^{0} \sin \left(\omega_{1} t\right), 0,0\right)$, of period $T_{1}$

$\left(0,0, X_{5}^{0} \cos \left(\omega_{2} t\right)+X_{6}^{0} \sin \left(\omega_{2} t\right), X_{6}^{0} \cos \left(\omega_{2} t\right)-X_{5}^{0} \sin \left(\omega_{2} t\right)\right)$, of period $T_{2}$.

Proof. Since (8) for $\varepsilon=0$ is a linear differential system the proof follows easily.

Proof of Theorem 1.1. Assume that the functions $F_{1}$ and $F_{2}$ of (1) are periodic in $t$ of period $p T_{1} / q$ with $p$ and $q$ positive integers relatively prime. Then, we can consider that the differential system (8) and the periodic solutions (9) have the same period $p T_{1}$.

We apply Theorem 4.1 of Appendix I to the differential system (8), and we use the notation introduced there. Note that system (8) can be written in the form of system (10) taking

$$
\begin{array}{r}
\mathrm{x}=\left(\begin{array}{l}
x_{1} \\
x_{2} \\
x_{3} \\
x_{4}
\end{array}\right), G_{0}(t, \mathrm{x})=\left(\begin{array}{c}
\omega_{1} x_{2} \\
-\omega_{1} x_{1} \\
\omega_{2} x_{4} \\
-\omega_{2} x_{3}
\end{array}\right), \\
G_{1}(t, \mathrm{x})=\left(\begin{array}{l}
F_{1}^{*} \\
F_{2}^{*} \\
F_{3}^{*} \\
F_{4}^{*}
\end{array}\right), G_{2}(t, \mathrm{x}, \varepsilon)=\left(\begin{array}{l}
0 \\
0 \\
0 \\
0
\end{array}\right)
\end{array}
$$

Now we shall study what periodic solutions of the unperturbed system (8) with $\varepsilon=0$ of the type (9) persist as periodic solutions for the perturbed one for $\varepsilon \neq 0$ sufficiently small.

We start with the description of the different elements which appear in the statement of Theorem 4.1 for the particular case of the differential system (8). Thus, we have that $\Omega=\mathbb{R}^{4}, k=2$ and $n=4$. Now, let $r_{1}>0$ be arbitrarily small and let $r_{2}>0$ be arbitrarily large. Let $V$ be the open and bounded subset of the plane $x_{3}=x_{4}=0$ of the form

$$
V=\left\{\left(X_{1}^{0}, X_{2}^{0}, 0,0\right) \in \mathbb{R}^{4}: r_{1}<\sqrt{\left(X_{1}^{0}\right)^{2}+\left(X_{2}^{0}\right)^{2}}<r_{2}\right\} .
$$

As usual $\mathrm{Cl}(V)$ denotes the closure of $V$. If $\alpha=\left(X_{1}^{0}, X_{2}^{0}\right)$, then we identify $V$ with the set $\left\{\alpha \in \mathbb{R}^{2}: r_{1}<\|\alpha\|<r_{2}\right\}$, being $\|\cdot\|$ the Euclidean norm in $\mathbb{R}^{2}$. The function $\beta: \mathrm{Cl}(\mathrm{V}) \rightarrow \mathbb{R}^{2}$ is $\beta(\alpha)=(0,0)$. Therefore, for our system we have

$$
\begin{aligned}
\mathscr{Z} & =\left\{\mathrm{z}_{\alpha}=(\alpha, \beta(\alpha)), \alpha \in \mathrm{Cl}(V)\right\}= \\
& =\left\{\left(X_{1}^{0}, X_{2}^{0}, 0,0\right) \in \mathbb{R}^{4}: r_{1} \leq \sqrt{\left(X_{1}^{0}\right)^{2}+\left(X_{2}^{0}\right)^{2}} \leq r_{2}\right\} .
\end{aligned}
$$

We are going to consider now, for each $\mathrm{z}_{\alpha} \in \mathscr{Z}$, the periodic solution $\mathrm{x}\left(t, \mathrm{z}_{\alpha}\right)=\left(X_{1}(t), X_{2}(t), 0,0\right)$ given by (9) of period $p T_{1}$.

Computing the fundamental matrix $M_{\mathrm{z}_{\alpha}}(t)$ of the linear differential system (8) with $\varepsilon=0$ associated to the 
$p T_{1}$-periodic solution $\mathrm{z}_{\alpha}=\left(X_{1}^{0}, X_{2}^{0}, 0,0\right)$ such that $M_{\mathrm{z}_{\alpha}}(0)$ be the identity of $\mathbb{R}^{4}$, we get

$$
\begin{aligned}
M_{\mathrm{z}_{\alpha}}(t) & =M(t)= \\
& =\left(\begin{array}{cccc}
\cos \left(\omega_{1} t\right) & \sin \left(\omega_{1} t\right) & 0 & 0 \\
-\sin \left(\omega_{1} t\right) & \cos \left(\omega_{1} t\right) & 0 & 0 \\
0 & 0 & \cos \left(\omega_{2} t\right) & \sin \left(\omega_{2} t\right) \\
0 & 0 & -\sin \left(\omega_{2} t\right) & \cos \left(\omega_{2} t\right)
\end{array}\right) .
\end{aligned}
$$

Note that the matrix $M_{\mathrm{z}_{\alpha}}(t)$ does not depend on the particular periodic solution $\mathrm{x}\left(t, \mathrm{z}_{\alpha}, 0\right)$. Since the matrix

$$
\begin{aligned}
M^{-1}(0)- & M^{-1}\left(p T_{1}\right)= \\
= & \left(\begin{array}{cccc}
0 & 0 & 0 & 0 \\
0 & 0 & 0 & 0 \\
0 & 0 & 2 \sin ^{2}\left(\frac{p \pi \omega_{2}}{\omega_{1}}\right) & \sin \left(\frac{2 p \pi \omega_{2}}{\omega_{1}}\right) \\
0 & 0 & -\sin \left(\frac{2 p \pi \omega_{2}}{\omega_{1}}\right) & 2 \sin ^{2}\left(\frac{p \pi \omega_{2}}{\omega_{1}}\right)
\end{array}\right)
\end{aligned}
$$

satisfies the assumptions of statement (ii) of Theorem 4.1 because the determinant

$$
\left|\begin{array}{cc}
2 \sin ^{2}\left(\frac{p \pi \omega_{2}}{\omega_{1}}\right) & \sin \left(\frac{2 p \pi \omega_{2}}{\omega_{1}}\right) \\
-\sin \left(\frac{2 p \pi \omega_{2}}{\omega_{1}}\right) & 2 \sin ^{2}\left(\frac{p \pi \omega_{2}}{\omega_{1}}\right)
\end{array}\right|=4 \sin ^{2}\left(\frac{p \pi \omega_{2}}{\omega_{1}}\right) \neq 0,
$$

because the ratio of the frequencies is non-resonant with $\pi$. In short, all the assumptions of Theorem 4.1 are satisfied by the system (8).

For our system the map $\xi: \mathbb{R}^{4} \rightarrow \mathbb{R}^{2}$ has the form $\xi\left(x_{1}, x_{2}, x_{3}, x_{4}\right)=\left(x_{1}, x_{2}\right)$. Calculating the function

$$
\begin{aligned}
\mathscr{G}_{1}\left(X_{1}^{0}, X_{2}^{0}\right) & =\mathscr{G}(\alpha)= \\
& =\xi\left(\frac{1}{p T_{1}} \int_{0}^{p T_{1}} M_{\mathrm{z}_{\alpha}}^{-1}(t) G_{1}^{1}\left(t, \mathrm{x}\left(t, \mathrm{z}_{\alpha}, 0\right)\right) d t\right),
\end{aligned}
$$

we obtain that

$$
\mathscr{G}_{1}\left(X_{1}^{0}, X_{2}^{0}\right)=\left(\mathscr{G}_{1}^{1}\left(X_{1}^{0}, X_{2}^{0}\right), \mathscr{G}_{1}^{2}\left(X_{1}^{0}, X_{2}^{0}\right)\right),
$$

where the functions $\mathscr{G}_{1}^{k}$, for $k=1,2$, are the ones given in (3). Then, by Theorem 4.1 we have that for every simple zero $\left(X_{1}^{0 *}, X_{2}^{0 *}\right) \in V$ of the system of nonlinear functions (4) we have a periodic solution $\left(x_{1}, x_{2}, x_{3}, x_{4}\right)(t, \varepsilon)$ of system (8) such that

$$
\left(x_{1}, x_{2}, x_{3}, x_{4}\right)(0, \varepsilon) \rightarrow\left(X_{1}^{0 *}, X_{2}^{0 *}, 0,0\right) \text { when } \varepsilon \rightarrow 0 .
$$

Going back through the change of coordinates (7) we get a periodic solution $\left(x_{1}, x_{2}, x_{3}, x_{4}\right)(t, \varepsilon)$ of system (8) such that

$\left(\begin{array}{c}x_{1}(t, \varepsilon) \\ x_{2}(t, \varepsilon) \\ x_{3}(t, \varepsilon) \\ x_{4}(t, \varepsilon)\end{array}\right) \rightarrow\left(\begin{array}{c}\frac{-2 c c_{2} \omega_{1}}{c_{2}+c^{2}(\rho-2 \phi)}\left(X_{2}^{0 *} \cos \left(\omega_{1} t\right)-X_{1}^{0 *} \sin \left(\omega_{1} t\right)\right) \\ c(\rho+2 \phi)\left(X_{1}^{0 *} \cos \left(\omega_{1} t\right)+X_{2}^{0 *} \sin \left(\omega_{1} t\right)\right) \\ \frac{-2}{\omega_{1}}\left(X_{2}^{0 *} \cos \left(\omega_{1} t\right)-X_{1}^{0 *} \sin \left(\omega_{1} t\right)\right) \\ 2\left(X_{1}^{0 *} \cos \left(\omega_{1} t\right)+X_{2}^{0 *} \sin \left(\omega_{1} t\right)\right)\end{array}\right)$

when $\varepsilon \rightarrow 0$.
Consequently we obtain a periodic solution $(x, y)(t, \varepsilon)$ of system (1) such that

$$
(x, y)(t, \varepsilon) \rightarrow\left(\begin{array}{c}
\frac{-2 c c_{2} \omega_{1}}{c_{2}+c^{2}(\rho-2 \phi)}\left(X_{2}^{0 *} \cos \left(\omega_{1} t\right)-X_{1}^{0 *} \sin \left(\omega_{1} t\right)\right) \\
\frac{-2}{\omega_{1}}\left(X_{2}^{0 *} \cos \left(\omega_{1} t\right)-X_{1}^{0 *} \sin \left(\omega_{1} t\right)\right)
\end{array}\right)
$$

when $\varepsilon \rightarrow 0$. This completes the proof of the theorem.

Proof of Theorem 1.3. The proof is analogous to the proof of Theorem 1.1 changing the roles of $T_{1}$ for $T_{2}$.

\section{Proof of the two corollaries}

Proof of Corollary 1.2. Under the assumptions of Corollary 1.2, the nonlinear system (4) becomes

$$
\begin{aligned}
\mathscr{G}_{1}^{1}\left(X_{1}^{0}, X_{2}^{0}\right)= & \frac{-X_{1}^{0} X_{2}^{0}(\rho+2 \phi)}{4 \rho} \\
\mathscr{G}_{1}^{2}\left(X_{1}^{0}, X_{2}^{0}\right)= & \frac{-2\left(1+\left(X_{1}^{0}\right)^{2}+3\left(X_{2}^{0}\right)^{2}\right) \phi}{8 \rho}- \\
& -\frac{\left(X_{1}^{0}\right)^{2}+3\left(X_{2}^{0}\right)^{2}-1}{8} .
\end{aligned}
$$

This system has the following four solutions

$$
\begin{aligned}
\left(X_{1}^{0 *}, X_{2}^{0 *}\right) & =\left(0, \pm \sqrt{\frac{1-c^{2} \phi(\rho-2 \phi)}{3}}\right), \\
\left(X_{3}^{0 *}, X_{4}^{0 *}\right) & =\left( \pm \sqrt{1-c^{2} \phi(\rho-2 \phi)}, 0\right) .
\end{aligned}
$$

Note that the solutions which differs in a sign are different initial conditions of the same periodic solution of the system (2). Moreover, since

$$
\left.\operatorname{det}\left(\frac{\partial\left(\mathscr{G}_{1}^{1}, \mathscr{G}_{1}^{2}\right)}{\partial\left(X_{1}^{0}, X_{2}^{0}\right)}\right)\right|_{\left(X_{1}^{0 *}, X_{2}^{0 *}\right)}=\frac{1}{4 c^{2} \rho^{2}} \neq 0
$$

and

$$
\left.\operatorname{det}\left(\frac{\partial\left(\mathscr{G}_{1}^{1}, \mathscr{G}_{1}^{2}\right)}{\partial\left(X_{1}^{0}, X_{2}^{0}\right)}\right)\right|_{\left(X_{3}^{0 *}, X_{4}^{0 *}\right)}=\frac{-1}{4 c^{2} \rho^{2}} \neq 0
$$

these solutions are simple. Finally, by Theorem 1.1 we only have two periodic solutions for the system of this corollary.

Proof of Corollary 1.4. Under the assumptions of Corollary 1.4, the nonlinear system (5) becomes

$$
\begin{aligned}
& \mathscr{G}_{2}^{1}\left(X_{5}^{0}, X_{6}^{0}\right)=-\frac{X_{5}^{0}}{2 c \rho}, \\
& \mathscr{G}_{2}^{2}\left(X_{5}^{0}, X_{6}^{0}\right)=-\frac{1+2 X_{6}^{0}}{4 c \rho} .
\end{aligned}
$$

This system has the following solution

$$
\left(X_{5}^{0 *}, X_{6}^{0 *}\right)=\left(0, \frac{-1}{2}\right) .
$$


Moreover, since

$$
\left.\operatorname{det}\left(\frac{\partial\left(\mathscr{G}_{2}^{1}, \mathscr{G}_{2}^{2}\right)}{\partial\left(X_{5}^{0}, X_{6}^{0}\right)}\right)\right|_{\left(X_{5}^{0 *}, X_{6}^{0 *}\right)}=\frac{1}{4 c^{2} \rho^{2}} \neq 0
$$

this solution is simple. Finally, by Theorem 1.3 we only have one periodic solution for the system of this corollary.

\section{Appendix: Basic results on averaging theory}

In this appendix we present the basic result from the averaging theory that we shall need for proving the main results of this paper.

We consider the problem of the bifurcation of $T$-periodic solutions from a differential system of the form

$$
\dot{\mathrm{x}}(t)=G_{0}(t, \mathrm{x})+\varepsilon G_{1}(t, \mathrm{x})+\varepsilon^{2} G_{2}(t, \mathrm{x}, \varepsilon),
$$

with $\varepsilon \neq 0$ sufficiently small. Here the functions $G_{0}, G_{1}: \mathbb{R} \times \Omega \rightarrow \mathbb{R}^{n}$ and $G_{2}: \mathbb{R} \times \Omega \times\left(-\varepsilon_{0}, \varepsilon_{0}\right) \rightarrow \mathbb{R}^{n}$ are $\mathscr{C}^{2}$ functions, $T$-periodic in the first variable, and $\Omega$ is an open subset of $\mathbb{R}^{n}$. The main assumption is that the unperturbed system

$$
\dot{\mathrm{x}}(t)=G_{0}(t, \mathrm{x}),
$$

has a submanifold of periodic solutions. A solution of this problem is given using the averaging theory.

Let $\mathrm{x}(t, \mathrm{z}, \varepsilon)$ be the solution of the system (11) such that $\mathrm{x}(0, \mathrm{z}, \varepsilon)=\mathrm{z}$. We write the linearization of the unperturbed system along a periodic solution $\mathrm{x}(t, \mathrm{z}, 0)$ as

$$
\dot{\mathrm{y}}=D_{\mathrm{x}} G_{0}(t, \mathrm{x}(t, \mathrm{z}, 0)) \mathrm{y} .
$$

In what follows we denote by $M_{\mathrm{z}}(t)$ some fundamental matrix of the linear differential system (12), and by $\xi: \mathbb{R}^{k} \times \mathbb{R}^{n-k} \rightarrow \mathbb{R}^{k}$ the projection of $\mathbb{R}^{n}$ onto its first $k$ coordinates; i.e. $\xi\left(x_{1}, \ldots, x_{n}\right)=\left(x_{1}, \ldots, x_{k}\right)$.

We assume that there exists a $k$-dimensional submanifold $\mathscr{Z}$ of $\Omega$ filled with $T$-periodic solutions of (11). Then an answer to the problem of bifurcation of $T$-periodic solutions from the periodic solutions contained in $\mathscr{Z}$ for system (10) is given in the following result.

Theorem 4.1. Let $V$ be an open and bounded subset of $\mathbb{R}^{k}$, and let $\beta: \mathrm{Cl}(V) \rightarrow \mathbb{R}^{n-k}$ be a $\mathscr{C}^{2}$ function. We assume that

(i) $\mathscr{Z}=\left\{\mathrm{z}_{\alpha}=(\alpha, \beta(\alpha)), \alpha \in \mathrm{Cl}(V)\right\} \subset \Omega$ and that for each $\mathrm{z}_{\alpha} \in \mathscr{Z}$ the solution $\mathrm{x}\left(t, \mathrm{z}_{\alpha}\right)$ of (11) is $T$-periodic;

(ii) for each $\mathrm{z}_{\alpha} \in \mathscr{Z}$ there is a fundamental matrix $M_{\mathrm{z}_{\alpha}}(t)$ of (12) such that the matrix $M_{\mathrm{z}_{\alpha}}^{-1}(0)-M_{\mathrm{z}_{\alpha}}^{-1}(T)$ has in the upper right corner the $k \times(n-k)$ zero matrix, and in the lower right corner a $(n-k) \times(n-k)$ matrix $\Delta_{\alpha}$ with $\operatorname{det}\left(\Delta_{\alpha}\right) \neq 0$.
We consider the function $\mathscr{G}: \mathrm{Cl}(V) \rightarrow \mathbb{R}^{k}$

$$
\mathscr{G}(\alpha)=\xi\left(\frac{1}{T} \int_{0}^{T} M_{\mathrm{z}_{\alpha}}^{-1}(t) G_{1}\left(t, \mathrm{x}\left(t, \mathrm{z}_{\alpha}, 0\right)\right) d t\right) .
$$

If there exists $a \in V$ with $\mathscr{G}(a)=0$ and $\operatorname{det}((d \mathscr{G} / d \alpha)(a)) \neq 0$, then there is a $T$-periodic solution $\mathrm{x}(t, \varepsilon)$ of system (10) such that $\mathrm{x}(0, \varepsilon) \rightarrow \mathrm{z}_{a}$ as $\varepsilon \rightarrow 0$.

For a proof of Theorem 4.1 see Malkin [11] and Roseau [12], or [1] for shorter proof.

\section{Acknowledgements}

This work has been partially supported by MCYT/FEDER grant number MTM2011-22587.

The authors want to express their acknowledgment to Prof. Juan Luis García Guirao and Prof. Juan Antonio Vera for their encouragement and guidance during the present research.

\section{References}

[1] A. Buica, J. P. Francoise and J. Llibre, Periodic solutions of nonlinear periodic differential systems with a small parameter, Comm. on Pure and Applied Analysis, 6, 103111 (2007).

[2] M. T. de Bustos, J. L. G. Guirao and J. A. Vera, The spatial hill lunar problem: periodic solutions emerging from equilibria, submitted.

[3] J. M. Ferrándiz, J. L. G. Guirao and J. A. Vera, A note on the periodic orbits of a self excited rigid body, submitted.

[4] J. L. G. Guirao, J. Llibre and J. A. Vera, Generalized van der Waals Hamiltonian: Periodic orbits and $C^{1}$ non-integrability, Physical Review E, 85, 1-5 (2012).

[5] J. L. G. Guirao, J. Llibre and J. A. Vera, Periodic solutions induced by an upright position of small oscillations of a sleeping symmetrical gyrostat, Nonlinear Dynamics, 73, 417-425 (2013).

[6] J. L. G. Guirao, J. Llibre and J. A. Vera, On the dynamics of the rigid body with a fixed point: periodic orbits and integrability, Nonlinear Dynamics, in press.

[7] J. L. G. Guirao, M. A. López and J. A. Vera, $C^{1}$ nonintegrability of a hydrogen atom in a circularly polarized microwave field, Central European Journal of Physics, 10, 742-748 (2012).

[8] J. L. G. Guirao, J. A. Vera and B. A. Wade, On the periodic solutions of a rigid dumbbell satellite in a circular orbit, Astrophysics and Space Science, 346, 437-442 (2013).

[9] J. Llibre and L. Roberto, On the periodic orbits of the third-order differential equation $x^{\prime \prime \prime}-\mu x^{\prime \prime}+x^{\prime}-\mu x=$ $\varepsilon F\left(x, x^{\prime}, x^{\prime \prime}\right)$, Applied Mathematics Letters, 26, 425-430 (2013).

[10] J. Llibre and L. Roberto, Periodic orbits and nonintegrability of Armbruster-Guckenheimer-Kim potential, Astrophysics and Space Science, 343, 69-74 (2013). 
[11] I. G. Malkin, Some problems of the theory of nonlinear oscillations, (Russian) Gosudarstv. Izdat. Tehn.-Teor. Lit., Moscow, (1956).

[12] M. Roseau, Vibrations non linéaires et théorie de la stabilité, (French) Springer Tracts in Natural Philosophy, SpringerVerlag, Berlin-New York, 8, (1966).

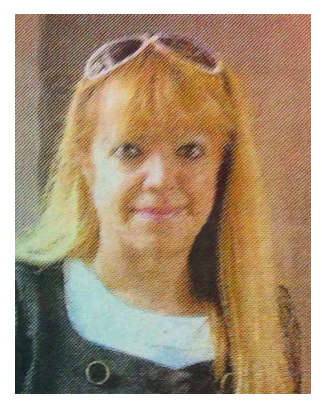

M. Teresa de Bustos is Associate Professor in the Department of Applied Mathematics at University of Salamanca in Spain, where she received the $\mathrm{PhD}$ degree in Mathematics. She has published some books and research articles in reputed international journals of mathematical and engineering sciences, and has presented some communications in prestigious international meetings. Her main research fields include dynamical systems, chaos theory, differential geometry and numerical methods for ODE.

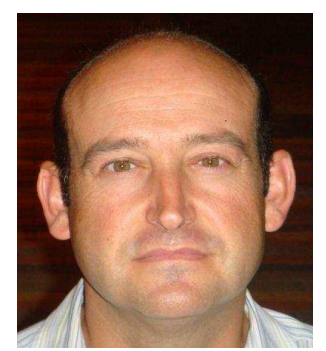

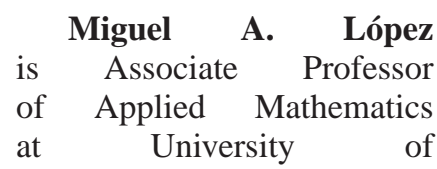
Castilla-La Mancha in Spain. He received the $\mathrm{PhD}$ degree in Mathematics at University of Murcia (Spain). He is an expert in dynamical systems and chaos theory. He mainly works in applied mathematics, engineering and mathematical physics problems. He has published many papers in high level research journals, he has advised two $\mathrm{PhD}$ thesis and he served as a Principal of the Polytechnic School of Cuenca (UCLM) for 8 years.

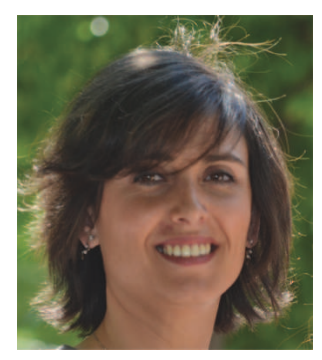

Raquel Martínez

in the Department of Applied

Mathematics at University

of Castilla-La Mancha

in Spain. She received the $\mathrm{PhD}$ degree in Mathematics at Universidad Politécnica de Cartagena (Spain). She has published some research articles in reputed international journals of mathematical and engineering sciences. Her main research interests are dynamical systems, chaos theory and analysis of the dynamics of systems with local competition "á la Cournot". 